\title{
Synthesis of Phospho-Amino Acid Analogues as Tissue Adhesive Cement Additives
}

\author{
Christopher D. Spicer,* Michael Pujari-Palmer, Hélène Autefage, Gerard Insley, Philip Procter, \\ Håkan Engqvist, and Molly M. Stevens*
}

Cite This: ACS Cent. Sci. 2020, 6, 226-231

Read Online

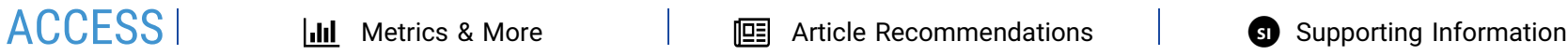

ABSTRACT: In this paper we report the synthesis of a library of phospho-amino acid analogues, via a novel single-step allylphosphoester protection/Pd-mediated deprotection strategy. These phosphoserine and phosphotyrosine analogues were then applied as additives to create adhesive calcium phosphate cements, allowing us to probe the chemical origins of the increased surface binding strength. We demonstrate the importance of multiple calcium binding motifs in mediating adhesion, as well as highlighting the crucial role played by substrate hydrophobicity and orientation in controlling binding strength.

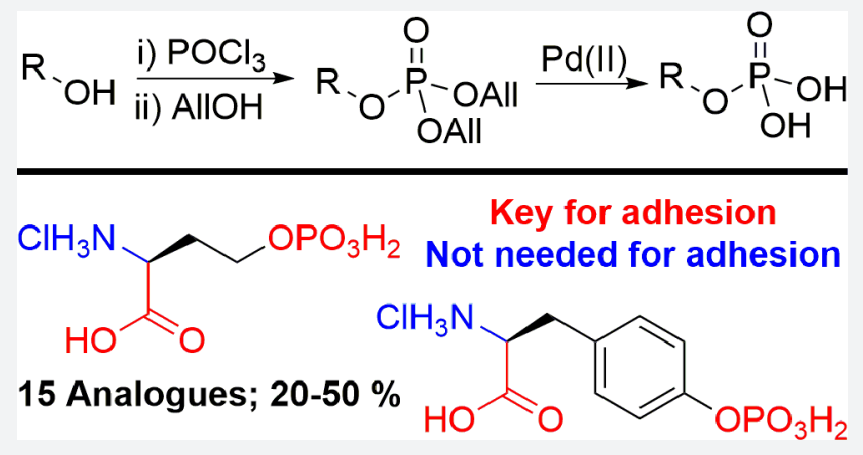

induces an organic/inorganic microstructure that templates calcium phosphate nucleation that may be responsible for the increased adhesion. ${ }^{13-15}$ PSer-modified cements (PMCs) are therefore able to produce strong biomaterial-tissue interfaces that enable their use within load-bearing environments. ${ }^{12,15}$ This has the advantage of enabling the use of CPCs as both fillers for bone defects and adhesive surfaces to form strong bridges between bone and alternative biomaterials such as metallic implants. Two commercial PMCs, Tetranite and OsStic, are currently undergoing advanced testing toward in vivo applications in dental and osteochondral applications. To investigate the ability of PSer to mediate adhesion, understand this phenomenon further, and potentially identify second generation additives able to enhance mechanical properties further, we set out to synthesize a library of phospho-amino acid analogues. The physicochemical properties of a cement are determined by the properties of the precursors, in the case of a PMC the organic amino acid and a basic calcium salt. We hypothesized that changes in the chemistry of the organic additive, for example through the removal of acidic or calcium chelating motifs, would directly affect the resulting adhesive strength. ${ }^{16,17}$ Mechanical (shear) testing would then be able to provide insight, at both the molecular- and macroscale, into the ex vivo relationship between additive chemistry and cement adhesive strength.

Received: November 7, 2019

Published: February 11, 2020 strength would reduce the likelihood of mechanical loosening. To address this problem, Kirillova et al. recently reported that phosphoserine (PSer, 1) can provide CPCs with remarkable adhesive strength. ${ }^{12}$ We have subsequently shown that PSer 
Scheme 1. Synthesis of Phospho-Tyrosine Analogues

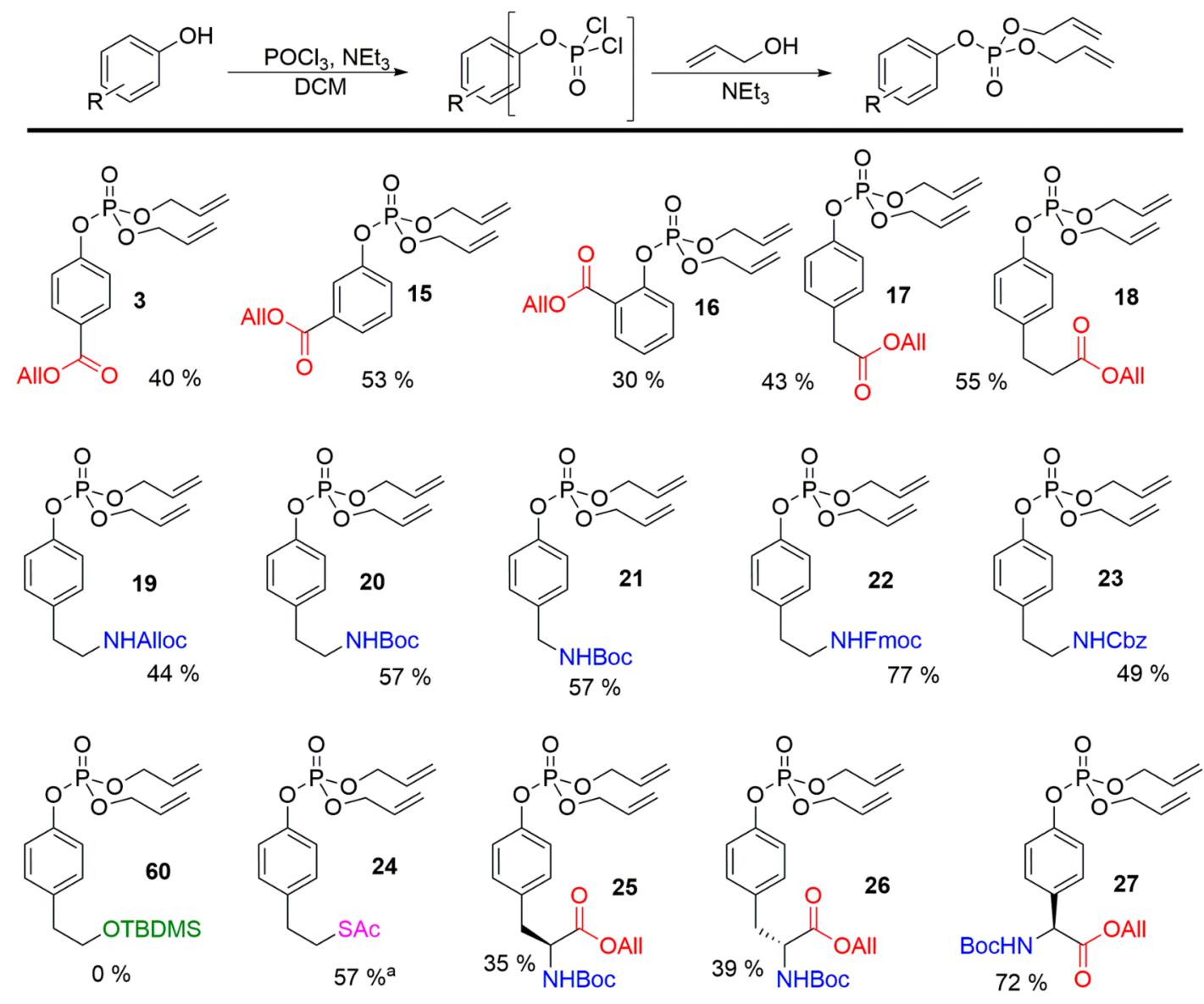

${ }^{a}$ Quenched with stoichiometric allyl alcohol.

The synthesis of phospho-amino acids has previously been achieved via three main approaches, each of which has limitations for library synthesis. In a first strategy, the desired phosphate group can be installed directly using a suitable phosphate donor. ${ }^{18,19}$ However, the resultant phosphoric acid monoesters are typically difficult to purify. ${ }^{20}$ Second, protected phosphonate esters can be installed through treatment with phosphorus $(\mathrm{V})$ oxychloride $\left(\mathrm{POCl}_{3}\right)$ and a sacrificial alcohol. The resultant phosphate esters are easily handled but typically require subsequent deprotection via basic hydrolysis. This challenging step must be carefully controlled to avoid competing phosphate cleavage. ${ }^{21}$ Finally, phosphoramiditebased reagents can be used to install protected-phosphite esters that must be subsequently oxidized to the corresponding phosphate. ${ }^{9}$ While this process allows orthogonal phosphate ester protecting groups to be introduced, the need for a second oxidative step with the potential for side reactions may be limiting. We therefore aimed to identify a global strategy for the introduction and deprotection of phosphate esters, which would also allow parallel and straightforward manipulation of the amino- and carboxy-protecting groups common in biomolecule substrates.

We initially envisaged the use of an acid-labile protecting group strategy through the installation of tert-butyl phosphate esters. $^{22}$ Although $\mathrm{O}^{t} \mathrm{Bu}$-phosphoramidite reagents were effective for alcohol phosphorylation, yields were often poor due to the high bulk of the $t \mathrm{Bu}$ ester. Attempts to utilize tertbutanol as a sacrificial alcohol following substrate treatment with $\mathrm{POCl}_{3}$ also proved ineffective. As an alternative, we reasoned that allyl protection would deliver an effective means to protect key functional groups during synthesis, and a convenient handle for palladium catalyzed deprotection. ${ }^{23}$ Furthermore, such an approach would be compatible with other commonly used protecting group strategies, including benzyl ethers and carbamates via orthogonal palladiumcatalyzed deprotection. ${ }^{24,25}$

4-Hydroxybenzoic acid allyl ester 2 was used as a model substrate as an analogue of tyrosine. After addition of $\mathbf{2}$ to a small excess of $\mathrm{POCl}_{3}$ and triethylamine (1.5 equiv each) and stirring for $1 \mathrm{~h}$, the reaction was quenched by addition of an excess of allyl alcohol and triethylamine. Pleasingly, clean conversion to the diallyl phosphate ester 3 was observed (Scheme 1). This procedure proved successful on a multigram scale, leading to the isolation of 3 in a $40 \%$ yield. The major impurities generated were found to be mixed allyl-hydroxy phosphates. Similar yields were obtained when the reaction was run under strictly anhydrous conditions or when no effort was made to exclude water or oxygen. This suggests that prior partial hydrolysis of the $\mathrm{POCl}_{3}$ was responsible for the generation of these side products. The use of fresh reagent was therefore important to maximize yields, though even old bottles of $\mathrm{POCl}_{3}$ (>1 year, stored under ambient atmosphere) delivered acceptable yields of 3 (40-60\%). We next applied our allyl-phosphate protection strategy to a series of tyrosineanalogue phenols. Allyl-protected carbonyls (2, 4-7) and alloc-protected amines (8) were well tolerated, as were 
Scheme 2. Synthesis of Phospho-Serine Analogues

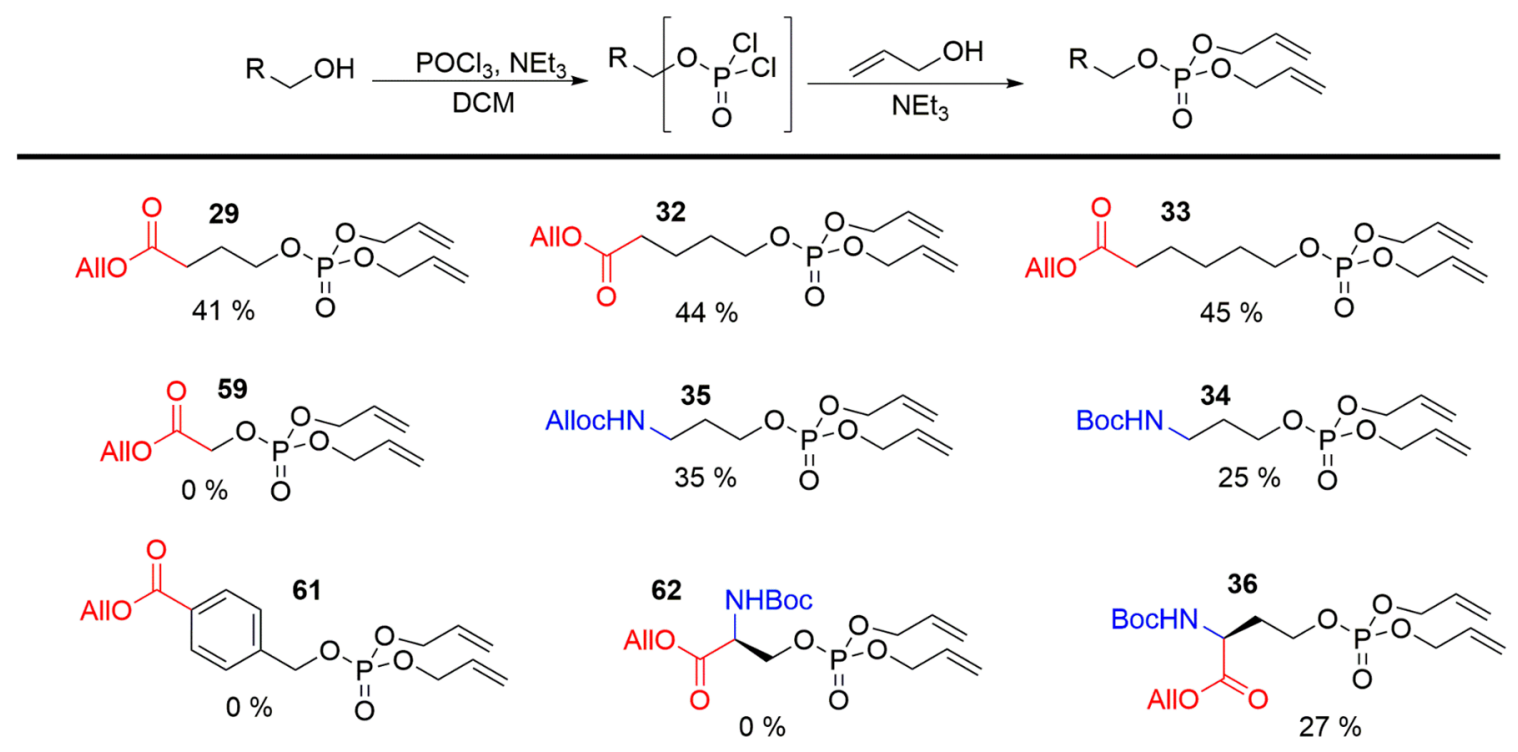

Scheme 3. Deprotection of Phospho-Amino Acid Library to Generate Phospho-Serine and Phospho-Tyrosine Analogues

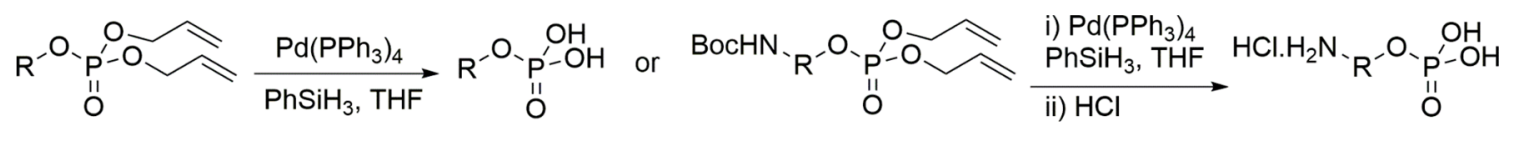<smiles>O=C(O)c1ccc(OP(=O)(O)O)cc1</smiles><smiles>O=C(O)c1cccc(OP(=O)(O)O)c1</smiles><smiles>O=C(O)c1ccccc1OP(=O)(O)O</smiles><smiles>O=C(O)Cc1ccc(OP(=O)(O)O)cc1</smiles><smiles>O=C(O)CCc1ccc(OP(=O)(O)O)cc1</smiles><smiles>NCc1ccc(OP(=O)(O)O)cc1</smiles><smiles>NCCc1ccc(OP(=O)(O)O)cc1</smiles><smiles>N[C@@H](Cc1ccc(OP(=O)(O)O)cc1)C(=O)O</smiles><smiles>N[C@@H](Cc1ccc(OP(=O)(O)O)cc1)C(=O)O</smiles><smiles>O=C(O)C([Al])c1ccc(OP(=O)(O)O)cc1</smiles><smiles>O=C(O)CCCOP(=O)(O)O</smiles><smiles>O=C(O)CCCOP(=O)(O)O</smiles><smiles>O=C(O)CCCCOP(=O)(O)O</smiles>

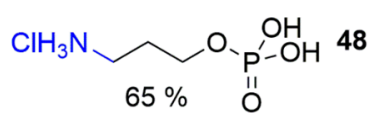<smiles>CN[C@@H](CCOP(=O)(O)O)C(=O)O</smiles>

alternative protecting groups including Boc- $(9,10)$, Fmoc(11), and benzyl- (12) amines. Ester-protected alcohols were stable under the reaction conditions; however, silyl ethers were poorly compatible (13). Protected thiols (14) were also compatible with the phosphorylation procedure, while orthofunctionalization was tolerated (5), albeit with a reduction in isolated yield. A library of protected phosphotyrosine (PTyr) analogues $(3,15-27)$ was therefore quickly generated from cheap and readily available reagents and intermediates.
Having demonstrated the use of our approach for phenol derivatization, we next switched our attention to the modification of alcohols. Due to the known propensity of $\beta$ hydroxyacids to undergo dehydration, particularly following phosphorylation, ${ }^{26}$ 4-hydroxybutanoic acid allyl ester 28 was used as a model substrate, as the deaminated analogue of phospho-homoserine. Under our optimized phosphorylation conditions, allyl phosphate 29 was delivered in a yield of $41 \%$ (Scheme 2). Boc- (30) and alloc- (31) protected amines were 
again well tolerated. No cyclization to the carbamate amine was isolated during the modification of $\mathbf{3 0}$ and $\mathbf{3 1}$, though in situ alcoholysis of an intermediate phosphoramidate cannot be discounted. ${ }^{27}$ A library of protected alcohols was readily generated $(29,32-36)$, including homoserine derivative 36 . The modifications of benzyl alcohol (37) and 2-hydroxyacetic acid (38) were ineffective, with chloride substitution of the alcohols instead being isolated as the major products. Furthermore, attempts to modify serine derivative 39 proved unsuccessful, with dehydroalanine $\mathbf{4 0}$ being formed as a result of dehydration.

With a library of protected PTyr and PSer analogues in hand, we then attempted to undertake allyl deprotection to yield the free phospho-derivatives. Palladium catalyzed deallylation is most commonly achieved in the presence of excess morpholine or a similar amine scavenger, that can react with the generated $\pi$-allyl palladium complex. The treatment of 3 with catalytic $\mathrm{Pd}\left(\mathrm{PPh}_{3}\right)_{4}$ and 10 equiv of morpholine led to rapid deprotection of both the phosphate- and carboxy-allyl esters. However, the use of a basic amine led to the product being isolated as the trimorpholine salt 41. Phenylsilane was therefore used as a nonbasic scavenger, leading to complete deprotection to form $\mathbf{4 2}$ in less than $20 \mathrm{~min}$. Pure $\mathbf{4 2}$ could be easily isolated in acidic form by partitioning between water and diethyl ether, and lyophilization of the aqueous fraction.

The developed deprotection conditions were able to deliver all members of the generated PTyr and PSer libraries (Scheme 3). Products commonly precipitated from solution as the reactions proceeded; however, yields were found to be reduced for analogues containing alloc-protected amines. While carbonyl- and phosphate-deallylation proceeded rapidly, alloc-deprotection was significantly slower. This resulted in the precipitation of partially deprotected impurities containing residual alloc-groups, lowering product yields. However, as these impurities were poorly soluble in water, purification could be achieved through simple filtration of the aqueous phase prior to lyophilization.

As an alternative approach, we also considered a 2-step deprotection strategy exploiting $\mathrm{N}$-Boc allyl phosphates. Bocprotected amine $\mathbf{2 0}$ was first treated under acidic conditions to generate free amino intermediate 43. Though subsequent deallylation was successful, the resultant amine was able to compete with phenylsilane as an allyl scavenger, leading to contamination of the final product with allyl-amine impurities. Reversing the order of deprotection, first undertaking deallylation and then acidic Boc removal proved more effective, generating the products $44-49$ as their hydrochloride salts (Scheme 3).

Having successfully synthesized a library of PSer and PTyr analogues $(\mathbf{4 2}, 44-56)$, they were next applied as additives within a CPC adhesion model, comparing adhesive (shear) strength as described in our previous report. ${ }^{13,14}$ Traditional CPCs display excellent bulk cohesive strength but poor adhesive strength, attaching to surfaces via weak electrostatic and van der Waals forces, and mechanical interdigitation. ${ }^{28}$ As recently reported by ourselves and Kirillova et al., PSer additives act to greatly enhance adhesion to many tissues and biomaterial surfaces, with exciting opportunities for applications in biomedicine. $^{12,13}$ However, the chemical origins of this effect remain unclear.

The adhesion of a $1 \mathrm{~cm}^{3}$ aluminum cube to a complementary cube was used to minimize substrate variation resulting from the use of heterogeneous tissue samples.
However, all results and trends subsequently discussed could be translated to the formation of bone-steel and bone-bone interfaces, as demonstrated through mechanical testing of a subset of analogues shown in the SI, Figure S1.

We began by comparing the adhesion of PMCs to cements containing other phospho-amino acids (Figure 1a). As
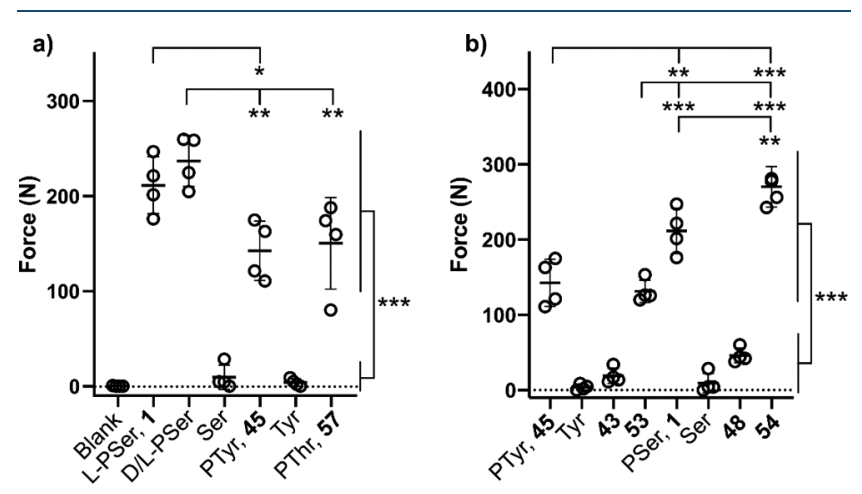

Figure 1. Comparison of cement adhesive strength in CPCs containing phospho-amino acid analogue additives. (a) Comparison across amino acids. (b) Comparison across analogues missing functional handles. Data are represented as scatter dot plots of each individual data point with the bars indicating the mean $\pm \mathrm{SD}$.* represents significant differences between groups $(* p<0.05$, ** $p<$ $0.005, * * * p<0.001)$. Details of the statistical analysis are provided in the SI.

previously observed, addition of PSer 1 to an $\alpha$-tricalcium phosphate CPC led to a large increase in adhesive strength $(211.6 \pm 30.1$ vs $0.2 \pm 0.4 \mathrm{~N})$. No significant difference was observed when either enantiopure or racemic PSer was used, supporting our hypothesis that adhesion is mediated by chemical rather than biological phenomena. PTyr 45 and phospho-threonine (PThr, 57) were also able to induce a similar increase in adhesive strength, albeit at a slightly reduced level (Figure 1a). Stereospecific conformational binding of PSer to the crystal surface of the CPC is therefore not essential to enable adhesion. Instead, the phospho-amino acid substrates share 4 chemical functionalities that may contribute to the observed adhesion: amine, carboxylic acid, and phosphate groups, and a carbon backbone. Furthermore, the steric arrangement of these functional groups is likely to play a critical role in dictating cement properties. We therefore set out to investigate the importance of each motif in isolation.

We hypothesized that multiple calcium binding motifs, arranged in a suitable orientation, would be required to induce adhesion (Figure 1b). Unsurprisingly, cements containing Lserine or L-tyrosine were poorly adhesive, highlighting the important role of the phosphate group. Similarly, decarboxylation of PTyr (analogue 43) resulted in cements with poor adhesive strength. However, the similarity in cement adhesion resulting from the addition of PTyr 45 and deaminated analogue $\mathbf{5 3}$ to the cement demonstrated that the amino functionality was dispensable. This observation was mirrored for PSer analogues, with deaminated phospho-homoserine analogue 54 mediating strong adhesion, in contrast to the drastic drop off in strength observed following the addition of decarboxylated 48. Cumulatively, these results support our hypothesis that monofunctional binding is insufficient for adhesion, with the amine motif being shown to not play an active role in bone binding. 
We next investigated the effect of carbon backbone length within our PSer series (Figure $2 \mathrm{a}$ ). We anticipated that changes
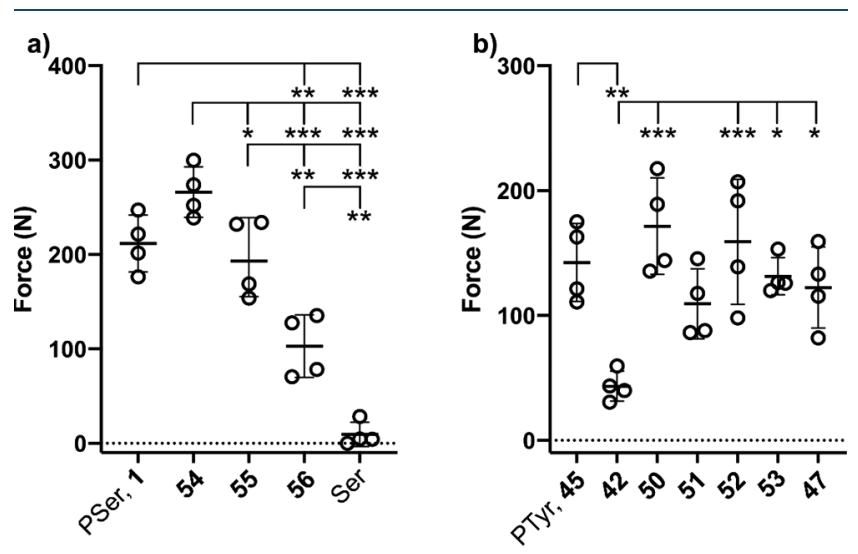

Figure 2. Comparison of cement adhesive strength in CPCs containing phospho-amino acid analogue additives. (a) Differences in adhesive strength between PSer analogues of increasing carbon chain length. (b) Differences in adhesive strength induced by phosphate-carboxylic acid orientation in PTyr analogues. Data are represented as scatter dot plots of each individual data point with the bars indicating the mean $\pm \mathrm{SD}$. * represents significant differences between groups $(* p<0.05$, ** $p<0.005$, *** $p<0.001)$. Details of the statistical analysis are provided in the SI.

in both the balance of amphiphilicity and the organization of the chelating motifs could lead to significant alterations in CPC properties. Indeed, we observed a gradual decrease in CPC adhesive strength when moving toward longer chain lengths (4-6 carbons, 54-56). Disappointingly, the instability of the 3-carbon analogue $\mathbf{5 8}$ noted earlier, and our inability to generate the corresponding 2-carbon analogue 59, prevented us from studying this effect further. However, the similarity in adhesive strength imparted by $\mathbf{5 4}$ and PSer suggests that no further increase would be induced by shorter chain lengths. Instead, the intriguing potential use of long chain phosphoacids as plasticizers warrants further investigation. 56, bearing a 6 carbon chain, was still able to provide notable adhesive strength when compared to L-serine.

Finally, we exploited the rigidity of the aromatic framework within our PTyr analogue library to investigate the role played by carboxyl-phosphate orientation (Figure $2 \mathrm{~b}$ ). Positioning of the phosphate group ortho $(\mathbf{5 1})$ or meta $(\mathbf{5 0})$ to the carboxylic acid led to CPCs with comparable adhesive strength to PTyr. In contrast, para-presentation of the phosphate group led to a significant drop in adhesion (analogue 42). This can be rationalized by the unfavorable orientation of the two calciumbinding motifs at opposite ends of the phenyl ring, suggesting that intra- rather than intermolecular bidentate binding is necessary for adhesion. This is supported by the observation that adhesive strength could be restored by extension of the carboxylic acid away from the ring system by as little as one carbon (52 and 53), providing flexibility that enables a favorable bidentate arrangement to again be achieved. Selected compounds (42, 50, 51, and PTyr 45) were also tested on freshly prepared bovine cortical bone cubes, with a higher sample size to account for large variability in tissue composition and surface roughness. The observed trends in bone (SI, Figure S1) matched those seen on metal substrates.

In conclusion, we have developed a novel allyl-protection strategy for phospho-amino acid synthesis, providing straight- forward access to a library of PSer and PTyr derivatives. This strategy allows the challenges associated with phosphate synthesis to be avoided, through late-stage global deprotection under mild and functional group-compatible Pd-mediated deallylation. The synthesized library could further be applied as additives in CPCs, to investigate the origins of PSer-induced cement adhesion. We have demonstrated that the presence of bidentate calcium binding motifs is necessary for high adhesive strength, with orientation and arrangement also playing a critical role. As this phenomenon does not require the presence of an amino motif, the possibility of condensation or polymerization reactions occurring during cement curing can be discounted. Collectively, these results demonstrate that CPCs can be transformed into adhesives by a wide array of heterobifunctional organophosphates. The clear trends observed during macroscale mechanical testing support the use of such a system to investigate differences in chemical structure at the molecular scale. We are currently investigating an expanded library bearing analogues with multidentate functional handles in an effort to better understand and enhance adhesive strength. With the increased mechanistic understanding provided by this work, we are also continuing to apply our PSer-containing cements in clinically relevant models of bone defect treatment. ${ }^{14}$

\section{ASSOCIATED CONTENT}

\section{Supporting Information}

The Supporting Information is available free of charge at https://pubs.acs.org/doi/10.1021/acscentsci.9b01149.

Synthesis details, spectral data of all new compounds, tabulated adhesive data, and full statistical analysis of significance (PDF)

\section{AUTHOR INFORMATION}

\section{Corresponding Authors}

Christopher D. Spicer - Department of Medical Biochemistry and Biophysics, Karolinska Institutet, 17177 Stockholm, Sweden; Department of Chemistry and York Biomedical Research Institute, University of York, Heslington YO10 5DD, United Kingdom; ○ orcid.org/0000-0001-8787-578X; Email: chris.spicer@york.ac.uk

Molly M. Stevens - Department of Medical Biochemistry and Biophysics, Karolinska Institutet, 17177 Stockholm, Sweden; Department of Materials, Department of Bioengineering, and Institute of Biomedical Engineering, Imperial College London, London SW7 2AZ, United Kingdom; (1) orcid.org/0000-00027335-266X; Email: m.stevens@imperial.ac.uk

\section{Authors}

Michael Pujari-Palmer - Applied Material Science, Department of Engineering, Uppsala University, 75121 Uppsala, Sweden

Hélène Autefage - Department of Medical Biochemistry and Biophysics, Karolinska Institutet, 17177 Stockholm, Sweden

Gerard Insley - Applied Material Science, Department of Engineering, Uppsala University, 75121 Uppsala, Sweden

Philip Procter - Applied Material Science, Department of Engineering, Uppsala University, 75121 Uppsala, Sweden

Håkan Engqvist - Applied Material Science, Department of Engineering, Uppsala University, 75121 Uppsala, Sweden

Complete contact information is available at:

https://pubs.acs.org/10.1021/acscentsci.9b01149 


\section{Notes}

The authors declare the following competing financial interest(s): M.P.-P., G.I., P.P., and H.E. declare partial ownership in a company that owns intellectual property related to PSer containing CPCs, GPBio LTD.

\section{ACKNOWLEDGMENTS}

C.D.S., M.P.-P., H.A., H.E., and M.M.S. acknowledge support from the Swedish Foundation for Strategic Research (SSF 4$3713 / 2296$ 2016). C.D.S. acknowledges support from the "Ulla och Gustaf af Ugglas Stiftelse". Raw data are available online at DOI: 10.5281 /zenodo.3648668.

\section{REFERENCES}

(1) Hunter, T. Why Nature Chose Phosphate to Modify Proteins. Philos. Trans. R. Soc., B 2012, 367 (1602), 2513-2516.

(2) Tsai, C. S. Biomacromolecules: Introduction to Structure, Function and Informatics; John Wiley \& Sons, Inc.: Hoboken, NJ, 2006.

(3) Helgadóttir, S.; Rosas-Sandoval, G.; Söll, D.; Graham, D. E. Biosynthesis of Phosphoserine in the Methanococcales. J. Bacteriol. 2007, 189 (2), 575-582.

(4) O'Leary, S. E.; Jurgenson, C. T.; Ealick, S. E.; Begley, T. P. OPhospho-L-Serine and the Thiocarboxylated Sulfur Carrier Protein CysO-COSH Are Substrates for CysM, a Cysteine Synthase from Mycobacterium Tuberculosis. Biochemistry 2008, 47 (44), 1160611615 .

(5) Connick, J. H.; Heywood, G. C.; Smith, D. A. S.; Stone, T. W. O-Phosphohomoserine, a Naturally Occurring Analogue of Phosphonate Amino Acid Antagonists, Is an N-Methyl-d-Aspartate (NMDA) Antagonist in Rat Hippocampus. Neurosci. Lett. 1986, 68 (2), 249251.

(6) Ma, C.; Tian, X.; Kim, J. P.; Xie, D.; Ao, X.; Shan, D.; Lin, Q.; Hudock, M. R.; Bai, X.; Yang, J. Citrate-Based Materials Fuel Human Stem Cells by Metabonegenic Regulation. Proc. Natl. Acad. Sci. U. S. A. 2018, 115 (50), E11741-E11750.

(7) Liang, P.; Zheng, J.; Zhang, Z.; Hou, Y.; Wang, J.; Zhang, C.; Quan, C. Bioactive 3D Scaffolds Self-Assembled from Phosphorylated Mimicking Peptide Amphiphiles to Enhance Osteogenesis. J. Biomater. Sci., Polym. Ed. 2019, 30 (1), 34-48.

(8) Ramaswamy, J.; Nam, H. K.; Ramaraju, H.; Hatch, N. E.; Kohn, D. H. Inhibition of Osteoblast Mineralization by Phosphorylated Phage-Derived Apatite-Specific Peptide. Biomaterials 2015, 73, 120130.

(9) Jessen, H. J.; Ahmed, N.; Hofer, A. Phosphate Esters and Anhydrides-Recent Strategies Targeting Nature's Favoured Modifications. Org. Biomol. Chem. 2014, 12 (22), 3526-3530.

(10) Xu, H. H. K.; Wang, P.; Wang, L.; Bao, C.; Chen, Q.; Weir, M. D.; Chow, L. C.; Zhao, L.; Zhou, X.; Reynolds, M. A. Calcium Phosphate Cements for Bone Engineering and Their Biological Properties. Bone Res. 2017, 5, 17056.

(11) Mann, K. A.; Miller, M. A.; Race, A.; Verdonschot, N. Shear Fatigue Micromechanics of the Cement-Bone Interface: An in Vitro Study Using Digital Image Correlation Techniques. J. Orthop. Res. 2009, 27 (3), 340-346.

(12) Kirillova, A.; Kelly, C.; von Windheim, N.; Gall, K. Bioinspired Mineral - Organic Bioresorbable Bone Adhesive. Adv. Healthcare Mater. 2018, 7, 1800467.

(13) Pujari-Palmer, M.; Guo, H.; Wenner, D.; Autefage, H.; Spicer, C. D.; Stevens, M. M.; Omar, O.; Thomsen, P.; Edén, M.; Insley, G.; et al. A Novel Class of Injectable Bioceramics That Glue Tissues and Biomaterials. Materials 2018, 11 (12), 2492.

(14) Procter, P.; Pujari-Palmer, M.; Hulsart-Billström, G.; Wenner, D.; Insley, G.; Larsson, S.; Engqvist, H. A Biomechanical Test Model for Evaluating Osseous and Osteochondral Tissue Adhesives. BMC Biomed. Eng. 2019, 1, 11.
(15) Liu, X.; Pujari-Palmer, M.; Wenner, D.; Procter, P.; Insley, G.; Engqvist, H. Adhesive Cements That Bond Soft Tissue Ex Vivo. Materials 2019, 12 (15), 2473.

(16) González Garcia, F.; Leyva, M. E.; Oliveira, M. G.; De Queiroz, A. A. A.; Simões, A. Z. Influence of Chemical Structure of Hardener on Mechanical and Adhesive Properties of Epoxy Polymers. J. Appl. Polym. Sci. 2010, 117 (4), 2213-2219.

(17) Feitosa, V. P.; Sauro, S.; Ogliari, F. A.; Ogliari, A. O.; Yoshihara, K.; Zanchi, C. H.; Correr-Sobrinho, L.; Sinhoreti, M. A.; Correr, A. B.; Watson, T. F.; et al. Impact of Hydrophilicity and Length of Spacer Chains on the Bonding of Functional Monomers. Dent. Mater. 2014, 30 (12), e317-e323.

(18) Dueymes, C.; Pirat, C.; Pascal, R. Facile Synthesis of Simple Mono-Alkyl Phosphates from Phosphoric Acid and Alcohols. Tetrahedron Lett. 2008, 49 (36), 5300-5301.

(19) Lira, L. M.; Vasilev, D.; Pilli, R. A.; Wessjohann, L. A. One-Pot Synthesis of Organophosphate Monoesters from Alcohols. Tetrahedron Lett. 2013, 54 (13), 1690-1692.

(20) Meziane, D.; Elias, A.; Guénin, E. Rapid and Efficient Synthesis of W-Alkylenediphosphoric Acids from Phosphorus Oxychloride. J. Chem. 2016, 2016, 8046893.

(21) Engel, R. Handbook of Organophosphorus Chemistry; CRC Press, 1992.

(22) Swarbrick, J. M.; Potter, B. V. L. Total Synthesis of a Cyclic Adenosine 5'-Diphosphate Ribose Receptor Agonist. J. Org. Chem. 2012, 77 (9), 4191-4197.

(23) Zhang, H. X.; Guibé, F.; Balavoine, G. Selective PalladiumCatalyzed Deprotection of the Allyl and Allyloxycarbonyl Groups in Phosphate Chemistry and in the Presence of Propargyl and Propargyloxycarbonyl Groups. Tetrahedron Lett. 1988, 29 (6), 623626.

(24) Vutukuri, D. R.; Bharathi, P.; Yu, Z.; Rajasekaran, K.; Tran, M.H.; Thayumanavan, S. A Mild Deprotection Strategy for AllylProtecting Groups and Its Implications in Sequence Specific Dendrimer Synthesis. J. Org. Chem. 2003, 68 (3), 1146-1149.

(25) Beugelmans, R.; Neuville, L.; Bois-Choussy, M.; Chastanet, J.; Zhu, J. Palladium Catalyzed Reductive Deprotection of Alloc: Transprotection and Peptide Bond Formation. Tetrahedron Lett. 1995, 36 (18), 3129-3132.

(26) Dadová, J.; Galan, S. R.; Davis, B. G. Synthesis of Modified Proteins via Functionalization of Dehydroalanine. Curr. Opin. Chem. Biol. 2018, 46, 71-81.

(27) Dujols, F.; Mulliez, M. The Influence of Ring Size on the Selectivity of Phosphorus Heterocycle Aminolysis in the Presence of Water or Alcohols - Case of 2-Oxo- or 2-Thioxo-3-Sulfonyl-1,3,2Oxazaphosphorinanes. Eur. J. Org. Chem. 2006, 2006 (8), 19591964.

(28) Grover, L. M.; Gbureck, U.; Farrar, D. F.; Barralet, J. E. Adhesion of a Novel Calcium Phosphate Cement to Cortical Bone and Several Common Biomaterials. Key Eng. Mater. 2006, 309-311, $849-852$. 